

\title{
ATTENUATION OUTER RETINAL BANDS ON OPTICAL COHERENCE TOMOGRAPHY FOLLOWING MACULAR EDEMA
}

Michel Paques, Florence Rossant, Lucia Finocchio, Kate Grieve, José-Alain Sahel, Alexandre Pedinielli, Sarah Mrejen

\section{To cite this version:}

Michel Paques, Florence Rossant, Lucia Finocchio, Kate Grieve, José-Alain Sahel, et al.. ATTENUATION OUTER RETINAL BANDS ON OPTICAL COHERENCE TOMOGRAPHY FOLLOWING MACULAR EDEMA: A Possible Manifestation of Photoreceptor Misalignment. RETINA. The Journal of Retinal and Vitreous Diseases, 2020, 40 (11), pp.2232-2239. 10.1097/IAE.0000000000002738 . hal-03025404

\section{HAL Id: hal-03025404 https://hal.sorbonne-universite.fr/hal-03025404}

Submitted on 26 Nov 2020

HAL is a multi-disciplinary open access archive for the deposit and dissemination of scientific research documents, whether they are published or not. The documents may come from teaching and research institutions in France or abroad, or from public or private research centers.
L'archive ouverte pluridisciplinaire HAL, est destinée au dépôt et à la diffusion de documents scientifiques de niveau recherche, publiés ou non, émanant des établissements d'enseignement et de recherche français ou étrangers, des laboratoires publics ou privés. 


\section{Attenuation outer retinal bands on optical coherence tomography following macular edema: a possible manifestation of photoreceptor misalignment}

Michel Paques ${ }^{1,2}, \mathrm{MD}, \mathrm{PhD}$, Florence Rossant ${ }^{3}, \mathrm{PhD}$, Lucia Finocchio ${ }^{1,4}, \mathrm{MD}$, Kate Grieve ${ }^{1}, \mathrm{PhD}$, José-Alain Sahel, $\mathrm{MD}^{1,2}$, Alexandre Pedinielli ${ }^{1}, \mathrm{MD}$, Sarah Mrejen ${ }^{1}, \mathrm{MD}$.

${ }^{1}$ Department of Ophthalmology, PARIS Group, Quinze-Vingts Hospital, INSERM-DGOS Clinical Investigation Center 1423, 28 rue de Charenton, F-75012 Paris, France

${ }^{2}$ Sorbonne Université, INSERM, CNRS, Institut de la Vision, 17 rue Moreau, F-75012 Paris, France

${ }^{3}$ Institut Supérieur d'Electronique de Paris, 75006 Paris, France

${ }^{4}$ Ophthalmology department, Carregi Hospital, Florence, Italy

Corresponding author: Michel Paques mpaques@15-20.fr

Acknowledgments: supported by the Agence Nationale de la Recherche (LabEx LifeSenses ANR-10-LABX-65, Recherche Hospitalo-Universitaire Light4Deaf 15-RHUS0001), the Foundation Fighting Blindness (C-GE-0912-0601-INSERM02), the French Clinical Research Initiative Network, the European Research Council (ERC-SyG 610110 Helmholtz) and the Association Contre l'OVR (asso-ovr.fr). The funding organization had no role in the design or conduct of this research.

Short title: Photoreceptor misalignement in macular edema

Presented in part at the 2016 meeting of the Association for Research in Vision and Ophthalmology (Seattle, WA)

Financial disclosure: MP and SM hold a patent (FR1153306) on multiangle OCT. None of the other authors has any financial interest to disclose.

Word count: 2558 
Keywords: photoreceptors, macular edema, optical coherence tomography, adaptive optics ophthalmoscopy, Stiles-Crawford effect.

Summary statement: In patients that recovered from macular edema, the presence of misaligned photoreceptors was evidenced by directional OCT and adaptive optics ophthalmoscopy. 
Purpose: Macular edema is a common retinal disease which may leave important anatomical and functional sequelaes. Directional fundus imaging consists of comparing on and off-axis images to reveal angle-dependent reflectance properties of fundus structures, which may be related to misaligned photoreceptors. Here we analyzed directional optical coherence tomography (OCT) and flood-illumination adaptive optics ophthalmoscopy (AOO) images in order to detect evidence of misaligned photoreceptors following macular edema.

Methods: Transversal, observational study. Nine patients having recovered a normal macular profile after macular edema due to retinal vein occlusion were included. For each patient, a reference OCT scan (i.e. with the incident beam normal to the fovea) was acquired, and off-axis scans were then acquired by laterally shifting the entry pupil. Additionally, in four of these eyes, directional $\mathrm{AOO}$ documented the directional variations of cone metrics.

Results: Included patients comprised 2 women and 7 men (age range, 19 to 76 years). Reference OCT scans showed patchy attenuation of the cone outer segment tips (COST) and to a lesser extent of the inner/outer segment (IS/OS) lines in all but two eyes; these. Increased intensity of the COST and IS/OS lines could be observed on off-axis scans. Accordingly, fusion images showed $66 \%$ average reduction of the length of COST attenuation. In two cases, while reference scans showed continuity of outer bands, focal attenuation was evidenced in off-axis images. Directional AOO imaging showed a strong directional variability of cone counts in these areas, ranging from near absence to roughly two-third of reference values. In each case directional variations of cone counts paralleled those of the reflectance of outer bands. .

Conclusions: Following macular edema, focal attenuations of the IS/OS and of the COST lines may be present on OCT. These areas may show a strong directional variability of by both OCT and AOO, suggesting that misaligned photoreceptors outer segments contribute to such feature. The evaluation of outer retinal damage following macular edema should therefore take into account the optical Stiles-Crawford effect in order to disambiguate missing from misaligned cones. 


\section{INTRODUCTION}

Optical coherence tomography (OCT) images are usually acquired in such a way that the OCT scan is horizontal on the live display screen, that is, with incident light normal to the retinal surface. Directional OCT (DOCT) involves deliberately imaging off-axis relative to this "standard" scan ${ }^{1-3}$. By varying the angle of incident light, directionally-sensitive areas of the retina demonstrate changes in reflectivity, hence providing additional optical contrast. The potential medical interest of DOCT was suggested by Lujan et al who used DOCT to extract the thickness of the outer nuclear layer ${ }^{4}$, taking advantage of the directional reflectance of the Henle fiber layer. Cone photoreceptors outer segments also show a strong directional variability of their reflectance, which is attributed to their waveguiding properties known as the optical Stiles-Crawford effect $(\mathrm{SCE})^{5}$. Cone show greater SCE than rods, possibly because diameter of the outer segment parallels waveguiding properties. The contribution of the photoreceptor substructures to the optical $\mathrm{SCE}^{6}$ was first analyzed by Gao et al. They suggested that directional reflectance of the cone outer segment tips (COST) line and, to a lesser extent, of the inner/outer segment junction (IS/OS) line ${ }^{7,8}$ (also termed interdigitation and ellipsoid zones ${ }^{9,10}$, respectively), accounts for the optical SCE of macular photoreceptors. The SCE enables to determine the overall orientation of outer segments. In normal eyes macular photoreceptors converge in most cases within $3 \mathrm{~mm}$ from the center of the cornea, often slightly nasally to the center, meaning that locally they can be considered as closely parallel. ${ }^{9-13}$ Using a combination of directional adaptive optics ophthalmoscopy (AOO) and DOCT, Miloudi et $\mathrm{al}^{14}$ documented the directional reflectance of normal photoreceptors and showed a correspondence between the directional variability of the COST and that of the cone mosaic.

These optical properties have been used to explore the orientation of outer segments in retinal diseases. In central serous chorioretinopathy ${ }^{15}$ and acute macular neuroretinopathy $(\mathrm{AMNR})^{16}$, directional reflectance of the outer retinal bands suggestive of photoreceptor misalignment have been reported. Finding such feature in two unrelated diseases suggests that photoreceptor misalignement may be rather common. Here, we explored the directional 
variability of photoreceptor imaging in a series of patients showing focal attenuation of outer retinal bands after having recovered from macular edema, which suggested the presence of misaligned photoreceptors.

\section{METHODS}

This observational study was carried out according to the tenets of the Declaration of Helsinki, and was approved by a french Comité de Protection des Personnes (reference 2017-A03223-50). Informed consent was obtained from all patients. The charts of 9 patients ( 2 women, 7 men) having recovered a normal OCT thickness profile but with persistence of patchy attenuation of the outer retinal bands after an episode of perfused macular oedema were selected. All had a standard ophthalmological examination. Eyes with choroidal hypertranmission suggestive of pigmentary changes, hemorrhages or exudates that could interfere with image interpretation were excluded. Their clinical characteristics are reported in table 1.

DOCT was performed using a combined scanning laser ophthalmoscope-OCT apparatus (Spectralis ${ }^{\circledR}$ SLO-OCT; Heidelberg Engineering, Heidelberg, Germany) according to a previously reported procedure ${ }^{16}$. Figure 1 shows a representative set of directional OCT scans in a normal eye. Briefly, a reference OCT scan was first acquired (either horizontal or vertical). This incidence appeared horizontal on the live image screen and the foveal pit showed a reflection spot located at its bottom; this incidence, which is normal to the retinal surface, is what we defined as the foveal axis. After tagging this image as the reference for the automatic registration procedure, off-axis OCT scans were captured by manually shifting the entry pupil laterally or vertically (i.e. in the same direction as the reference scan) using the follow-up mode, while the patient maintained fixation. For each reference scan, 6 to 10 off-axis scans were acquired with stepwise increase in eccentricities on each side. The varying positions of the foveal spot demonstrated that there actually was an angular shift of incident light relative to the foveal axis.

Registered OCT scans were checked offline to ensure the accuracy of the registration process, which was considered acceptable if there were no obvious changes in the pattern of 
choroidal vessels. On off-axis images, the COST lines and, to a lesser extent, the IS/OS showed a general attenuation (example in figure 1), in accordance with previous reports ${ }^{4}$.

To measure the angular shift and hence ensure comparability with directional AOO incidences, we took advantage of the fact that the registration process generates a blind (dark) triangle in a corner of the registered image which is the geometrical illustration of the angular shift relative to the reference scan ${ }^{17}$. Hence, the actual change in light incidence relative to the foveal axis is given by the value of the acute angle adjacent to the superior border of the scan. OCT scans taken between 1.5 and $2.5^{\circ}$ eccentricity were selected for further analysis. These values were chosen because similar eccentricities were obtained by AOO (see below).

The foveal thickness, the OS length and the ONL thickness (after exclusion of HFL) were measured manually. The ONL was defined as the distance between the outer limit of the HFL and the external limiting membrane; the OS length was defined as the center of the IS/OS to the outer border of the COST. To ensure comparability of measures between affected and nonaffected areas, for each affected area an area symmetrically placed to the fovea was considered as the control zone. Fusion images were constructed using ImageJ. The relative decrease in the length of attenuation of the COST (measured along a $3 \mathrm{~mm}$ length centered on the fovea, except for case 7 who showed directional variability in the paramacular area) was calculated.

In four eyes, directional flood-illumination $\mathrm{AOO}$ was performed ( $\mathrm{rtx} 1^{\mathrm{TM}}$ camera, Imagine Eyes, Orsay, France) using a slightly modified procedure breviously described ${ }^{14}$. Briefly, the field of view was placed over an area showing directional variability by OCT. A reference image in which the illumination beam (identified by a cross) and the corneal apex (identified by corneal reflections) were aligned was acquired. For off-axis imaging, adjusting the corneal reflection dots relative to the center of the beam enabled to place the incident beam 1.5 to $2.5^{\circ}$ off-axis $^{14}$. Although imaging at $2.5^{\circ}$ eccentricity was attempted in every case, the final excentration was chosen based on the excentration that enabled the best contrast on the live 
screen within the 1.5 to $2.5^{\circ}$ limits. This procedure was repeated in the cardinal directions. This resulted in a set of five images (one on-axis and four shifted in the cardinal directions) for each region of interest $(\mathrm{ROI})$. $\mathrm{AOO}$ images of the same $\mathrm{ROI}$ were registered using $\mathrm{i} 2 \mathrm{k}$ Align Retina software (DualAlign, LLC, Clifton Park, NY). Unsupervised photoreceptors counts were done on $100 \times 100 \mu m$ areas by the built-in software of the rtx 1 camera (AO detect $)^{18}$ on aligned images. On AOO images taken off-axis, the cone mosaic showed variably decreased values (example in figure 2). Reference values for cone densities were from ${ }^{19,20}$. The directional variability of cone counts in healthy eyes using this procedure is shown in supplementary figure 1.

\section{RESULTS}

There were 2 females and 7 males, with a mean age ( \pm SD) of 43.1 years; age range 1976). The examination was done at a mean of 10.2 months after the vein occlusion episode (range, 2-36). Five eyes had received intravitreal injections of anti-vascular endothelial growth factor. LogMAR visual acuity (VA) ranged from +0.2 to $0(20 / 32$ to $20 / 20)$. The foveal thickness was similar between affected and fellow eyes (mean \pm SD $212 \mu \mathrm{m} \pm 22$ and 236 $\mu \mathrm{m} \pm 26$ respectively; $p=0.23$ )

On reference scans of affected eyes, 7 out of 9 showed patchy attenuation of the COST and of the IS/OS lines (examples in figure 3). In all cases the COST was more attenuated than the IS/OS. The external limiting membrane and the retinal pigment epithelium appeared normal in all cases. These changes affected foveal photoreceptors in 6 cases, which included the three cases with reduced VA. A single area with attenuated COST was detected in 4 cases, and two or three attenuated areas were detected in 5 other cases. The size of these attenuated areas varied from $\sim 200 \mu \mathrm{m}$ to $\sim 1 \mathrm{~mm}$. Overall the OS length and ONL thickness (after exclusion of the HFL) were nonsignificantly different in attenuated and adjacent areas (Table 1). Most of these attenuated zones increased the reflectance of their COST and IS/OS lines in one off-axis image (figure 4). In most case, the IS/OS and the COST showed parallel changes, that is, they increased their reflectance on the same off-axis image. In two case (6 and 9), while outer bands were normal in the reference image, the 
presence of an area with attenuated COST line was seen on off-axis images (Figure 4, right column). Accordingly, fusion OCT images (i.e. display of the pixels with the highest grayscale value among registered scans) showed improvement of the continuity of outer retinal bands in the 7 case with attenuated COST on the reference image (figure 5 and supplementary figure 1). Overall, in fusion images the apparent length of the attenuated COST area decreased on average by $66 \%$.

Such attenuation of outer bands affected the fovea in 4 eyes. A slight improvement of the intensity of foveal outer bands on off-axis scans was seen in two of these (cases 3 and 8). In case 3, both the COST and the IS/OS increased their reflectance (see figure 4, bottom left), while in case 8 , the IS/OS increased its relative reflectance but changes of the foveal COST line were less clear.

Directional $\mathrm{AOO}$ was done in four cases. On on-axis $\mathrm{AOO}$ images, the cone mosaic was dim or absent in all areas showing attenuated COST. Comparing off-axis images showed that the contrast of the cone mosaic was increased in at least one direction (figures 6 and 7). This direction was in all case the same that gave the highest contrast of the IS/OS and COST by OCT, that is, the direction relative to the center of the cornea giving the highest contrast of the COST also led to the highest contrast of the cone mosaic. Accordingly, there was a strong directional variability of cone counts (table 2), ranging from near zero to roughly two-third of reference values.

\section{DISCUSSION}

In a series of eyes that had recovered from macular edema we observed the presence of patchy attenuations of outer retinal bands in the macula. Here we investigated the hypothesis that misaligned photoreceptors could account for such feature. We found that in all these eyes, most areas with attenuated outer retinal bands showed a clear directional modulation of their reflectance, strongly suggesting the presence of waveguiding cones. Accordingly, the width of attenuated areas was significantly reduced on fusion images. The directional changes of outer retinal bands paralleled the visibility of the cone mosaic by directional AOO. This renders unlikely vitreous floaters as the cause of such attenuation. 
The optical SCE, a manifestation of waveguiding properties of cone photoreceptors, is commonly assumed to be related to the physical orientation of the outer segment of cone photoreceptors, with the peak intensity of cones occurring when incident light is coaxial to outer segments. Our findings therefore argue for the hypothesis that attenuated areas comprised misaligned outer segments. Our conclusions are similar to those of previous reports supporting the presence of misaligned photoreceptors ouster segments following central serous chorioretinopathy ${ }^{15}$ and $\mathrm{AMNR}^{16}$.

The fact that in most cases both the IS/OS and COST showed attenuation may imply that both inner and outer segments were misaligned. The attenuation was nevertheless more important on the COST than on the IS/OS which may be related to the fact that both rods and cones contribute to the intensity of the IS/OS hence the rod contribution, which is less effected by the SCE, is relatively preserved. Parallel misalignment of several adjacent photoreceptors is understandable if one considers that outer segments are packed one to the other and have therefore limited possibility to diverge. This may be in particular seen if there is no loss of photoreceptors, meaning that "outer segment crowding" can exert its full effect. Outer segments may therefore show coordinated misplacement whenever there is weakening of the attachment to the RPE. In such situation, outer segments orientate according to the mainstream forces resulting from the deformation of the retina and/or the presence of circulating fluids, among possible factors. This does not exclude the possibility that individual photoreceptors may divert from this general misorientation; individual divergences may be commensurate to loss of outer segments hence lessening the crowding effect. Modifications of the extracellular space surrounding outer segments may also account for individual deviations.

Among possible mechanisms accounting from such misalignment are changes in the Muller cells architecture (which have been suggested to have a waveguiding effect ${ }^{21}$ ), in the $\mathrm{RPE} /$ photoreceptor junction or of the volume of the interphotoreceptor matrix. We noted a decreased density of cones in the affected areas. The loss of rods could also theoretically increase the volume of the extracellular space and hence modify the mechanical support of 
outer segments. Compared to SLO based systems, our flood illumination AOO system has the advantage of a relatively large depth of field hence reducing the effect of defocus. Therefore, inaccuracy of focusing was unlikely to be the cause of directional variations. Additionally, there were surrounding cones clearly visible in each AOO image, suggesting adequate focus. However, a disadvantage of flood-based $\mathrm{AOO}$ systems compared to scanning systems is that the resolution is slightly inferior, and that it cannot detect cones that do not possess an outer segment (non-waveguiding cones), which are electively detected by split-detection AOO. Therefore, we cannot ensure that the peak density we measured in the attenuated areas indeed corresponds to the whole population of cones. Indeed, outer segments with a higher angulation and/or cone without outer segments may have escaped detection. Hence, we cannot exclude the loss of photoreceptors as a contributor to cone misalignment.

Directional high resolution imaging is contributing to a better knowledge of the phenotype of diseased cones. Multimodal directional imaging indeed helped to solve some findings such as those reported in a MacTel case in which photoreceptor function was present in area without detectable cones by on-axis AOSLO and $\mathrm{OCT}^{21}$. Among other possibilities, this may be due to misaligned cones, as we previously reported such evidence during MacTel (Pedinielli et al: Photoreceptor anisotropy in macular oedema and MacTel : a multiangle OCT study. Invest Ophthalmol Vis Sci 2016, Vol.57, ARVO abstract 4248). It may nevertheless also be related to the recently described dysflective cones ${ }^{20}$ which relates to the persistence of light perception in the absence of detectable cone inner or outer segments, suggesting that severely remodeled outer segments may nevertheless yield visual function.

To sum up, patients that had macular edema may present patches of photoreceptor misalignment that can manifest by focal attenuation of outer retinal bands. Some of these misaligned photoreceptors may be detected only on off-axis scans. Therefore, attenuated reflectance of outer bands is not necessarily indicative of a decreased amount of cone outer segments. The evaluation of outer retinal damage following macular edema should therefore take into account the directional variability of photoreceptors imaging in order to 
disambiguate missing from misaligned cones. Multimodal directional imaging is of particular interest to ascertain these subtle yet potentially functionally important changes, since photoreceptor pointing modulates the visual efficacy of cones ${ }^{9}$. To explore the functional consequences of photoreceptor misalignment, comparing retinal sensitivity through different entry pupils may be of interest.

\section{REFERENCES}

1. Lujan BJ, Roorda A, Knighton RW, Carroll J. Revealing Henle's fiber layer using spectral domain optical coherence tomography. Invest Ophthalmol Vis Sci. 2011,52:14861492.

2. Otani T, Yamaguchi $\mathrm{Y}$, Kishi S. Improved visualization of Henle fiber layer by changing the measurement beam angle on optical coherence tomography. Retina. $2011 ; 31: 497-501$

3. Mrejen S, Gallego-Pinazo R, Freund KB, Paques M. Recognition of Henle's fiber layer on OCT images. Ophthalmology. 2013;120:e32-3.e1.

4. Lujan BJ, Roorda A, Croskrey JA et al. Directional optical coherence tomography provides accurate outer nuclear layer and Henle fiber layer measurements. Retina. 2015;35:1511-1520.

5. Westheimer G. Directional sensitivity of the retina: 75 years of Stiles-Crawford effect. Proc Biol Sci. 2008;275:2777-2786.

6. Gao W, Cense B, Zhang $Y$ et al. Measuring retinal contributions to the optical StilesCrawford effect with optical coherence tomography. Opt Express. 2008;16:6486-6501.

7. Jonnal RS, Kocaoglu OP, Zawadzki RJ et al. The cellular origins of the outer retinal bands in optical coherence tomography images. Invest Ophthalmol Vis Sci. 2014;55:79047918.

8. Jonnal RS, Gorczynska I, Migacz JV et al. The Properties of Outer Retinal Band Three Investigated With Adaptive-Optics Optical Coherence Tomography. Invest Ophthalmol Vis Sci. 2017;58:4559-4568. 
9. Spaide RF, Curcio CA. Anatomical correlates to the bands seen in the outer retina by optical coherence tomography: literature review and model. Retina. 2011;31:1609-1619.

10. Litts KM, Zhang Y, Freund KB, Curcio CA. Optical coherence tomography and histology of age-related macular degeneration support mitochondria as reflectivity source. Retina. 2018;38:445-461.

11. Burns SA, Wu S, Delori FC, Eisner AE. Direct measurement of human cone photoreceptor alignment. J Opt Soc Am A. 1995;12:2329-2338.

12. Roorda A, Williams DR. Optical fiber properties of individual human cones. J Vis. 2002;2:404-412.

13. Morris HJ, Blanco L, Codona JL et al. Directionality of individual cone photoreceptors in the parafoveal region. Vision Res. 2015;117:67-80.

14. Miloudi C, Rossant F, Bloch I et al. The Negative Cone Mosaic: A New Manifestation of the Optical Stiles-Crawford Effect in Normal Eyes. Invest Ophthalmol Vis Sci. 2015;56:7043-7045

15. Sun LW, Carroll J, Lujan BJ. Photoreceptor disruption and vision loss associated with central serous retinopathy. Am J Ophthalmol Case Rep. 2017;8:74-77.

16. Bottin C, Grieve K, Rossant F et al. Directional variability of fundus reflectance in acute macular neuroretinopathy: evidence for a contribution of the Stiles-Crawford effect. Retinal Cases and Brief Reports. 2018;12:S19-S24.

17. Park D, Lujan, BJ. Normal interdigitation zone loss by motion-tracked OCT. Ophthalmology Retina 2017;1: 394

18. Lazareva A, Liatsis P, Rauscher FG. Hessian-Log Filtering for Enhancement and Detection of Photoreceptor Cells in Adaptive Optics Retinal Images. Journal of the Optical Society of America A 2015;33:84-94.

19. Jacob J, Paques M, Krivosic V et al. Comparing Parafoveal Cone Photoreceptor Mosaic Metrics in Younger and Older Age Groups Using an Adaptive Optics Retinal Camera. Ophthalmic Surg Lasers Imaging Retina. 2017 ; 48:45-50 
20. Legras, R., Gaudric, A., Woog, K. Distribution of Cone Density, Spacing and Arrangement in Adult Healthy Retinas with Adaptive Optics Flood Illumination. PLoS one 201813, e0191141.

21. Zueva L, Makarov V, Zayas-Santiago A et al. Müller cell alignment in bird fovea: possible role in vision. J Neurosci Neuroeng. 2014;3: 85-91.

22. Tu JH, Foote KG, Lujan BJ et al. Dysflective cones: Visual function and cone reflectivity in long-term follow-up of acute bilateral foveolitis. Am $\mathrm{J}$ Ophthalmol Case Rep. 2017;7:14-19.

23. Wang Q, Tuten WS, Lujan BJ et al. Adaptive optics microperimetry and OCT images show preserved function and recovery of cone visibility in macular telangiectasia type 2 retinal lesions. Invest Ophthalmol Vis Sci. 2015 13;56:778-86.

\section{Legend to figures}

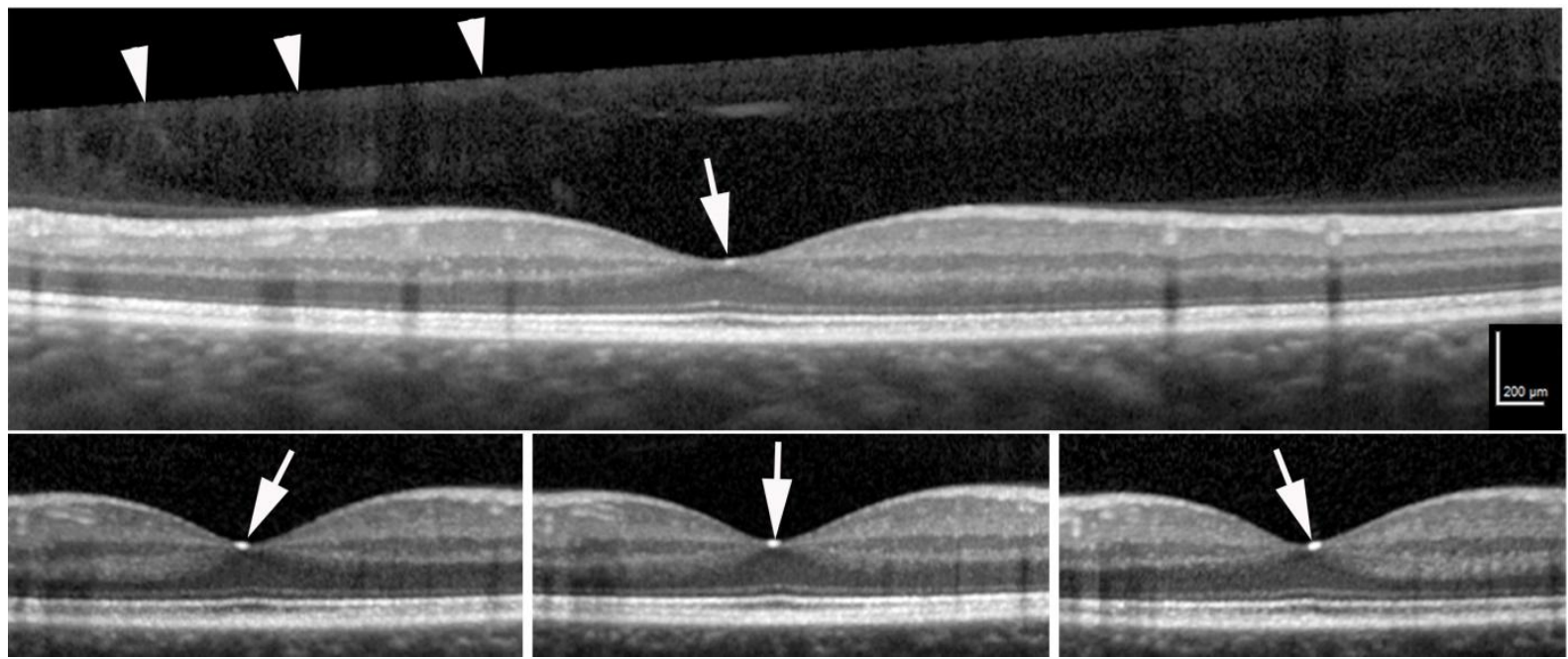

Figure 1. DOCT in a normal eye. Top, the spot in the foveal pit (arrow) indicates the direction of incident light, which is normal to the tangent at that point and to the hypotenuse (arrowheads) of the black triangle in the upper left corner. Bottom, OCT scans taken on-axis (middle) and off-axis. Arrows show the foveal spot. Note the overall decrease of the intensity of the IS/OS and COST bands on off-axis images. In subsequent figures, arrows in foveal pit point the foveal spot and are oriented normal to the retinal surface, that is, parallel to incident light. 

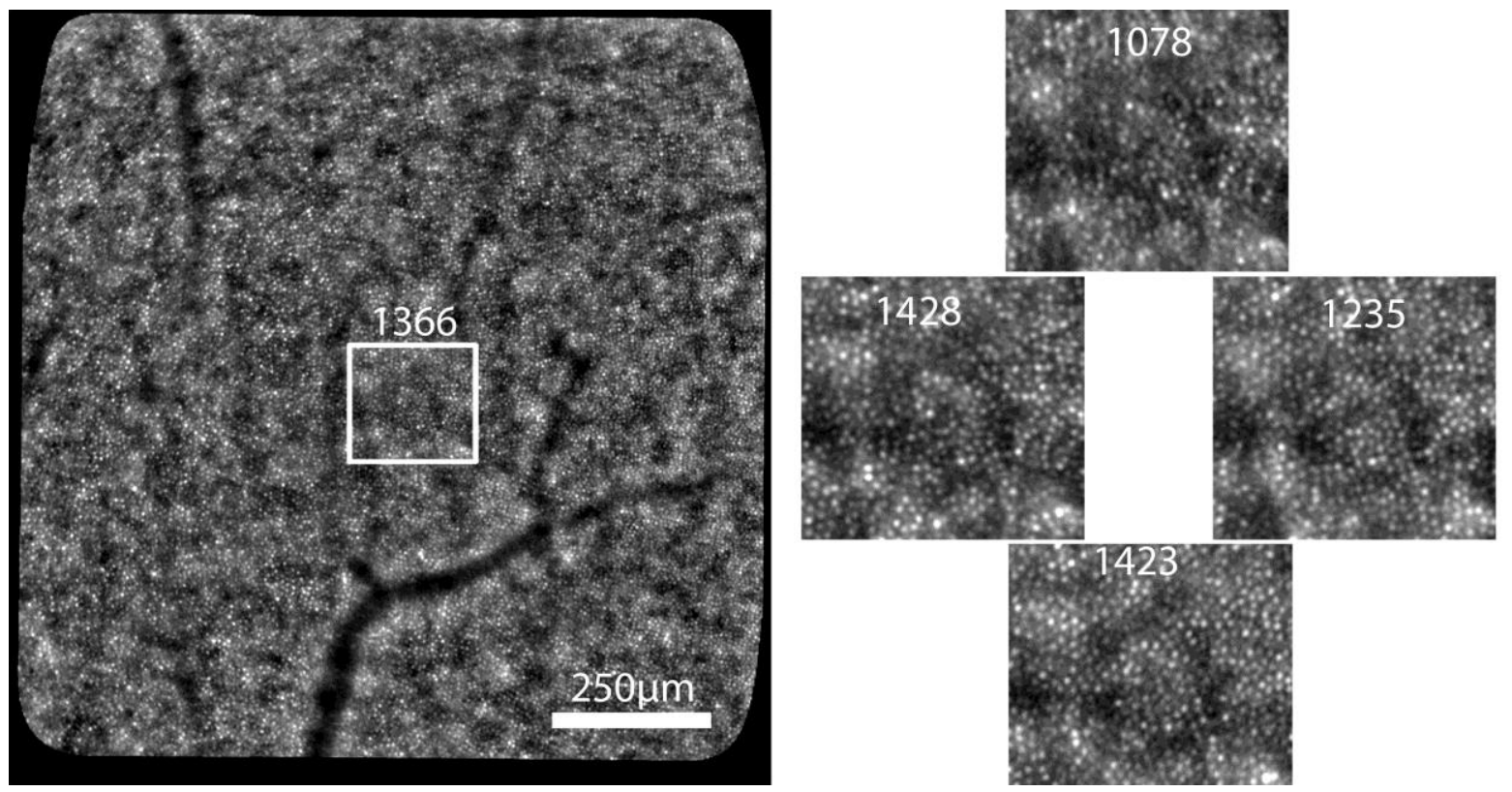

Figure 2. Directional AOO in a normal eye (same eye as in figure 1). Images were taken $4^{\circ}$ temporal. Left, on-axis image. The square shows the area in which automated cone counts were done. Right, off-axis images of the same area. The cone density $\left(/ \mathrm{min}^{2}\right)$ are indicated.
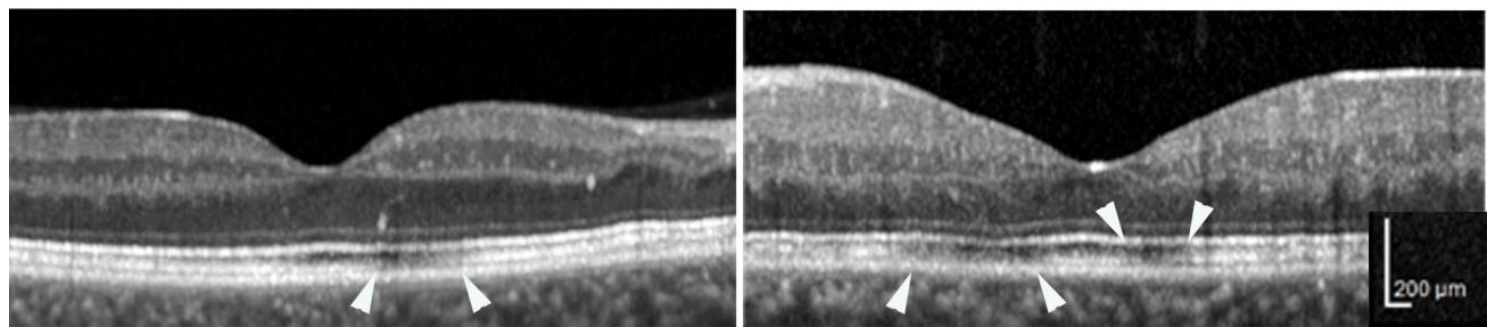

Figure 3. Reference OCT scans of cases 1 (left) and 2 illustrating the patchy attenuations of the COST and IS/OS lines (between arrowheads). 

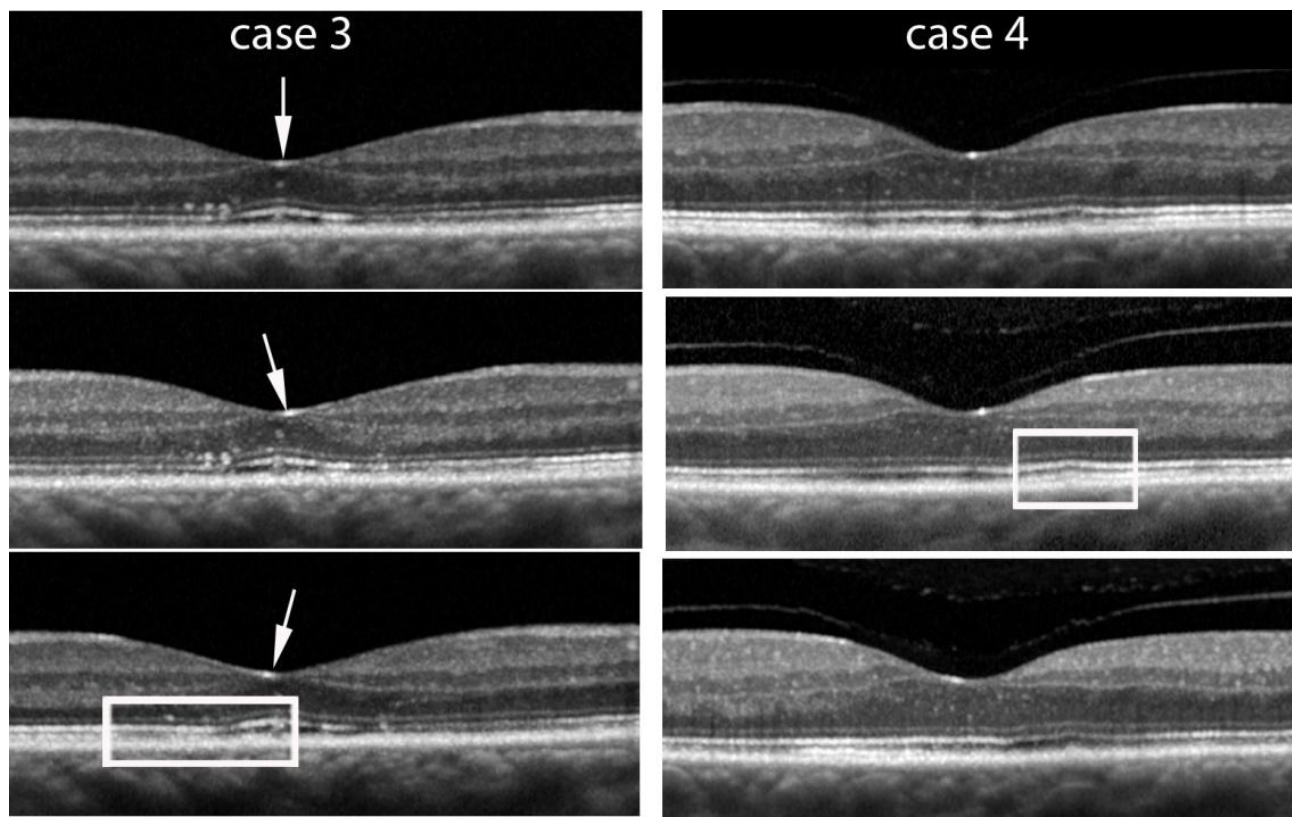

Figure 4. DOCTs of cases 3 and 4 illustrating directional variability of outer retinal bands. Upper row : reference scans; middle row and bottom rows: OCT scan taken off-axis: In the rectangles are examples of areas showing increased reflectance of the IS/OS and COST relative to the reference scan.
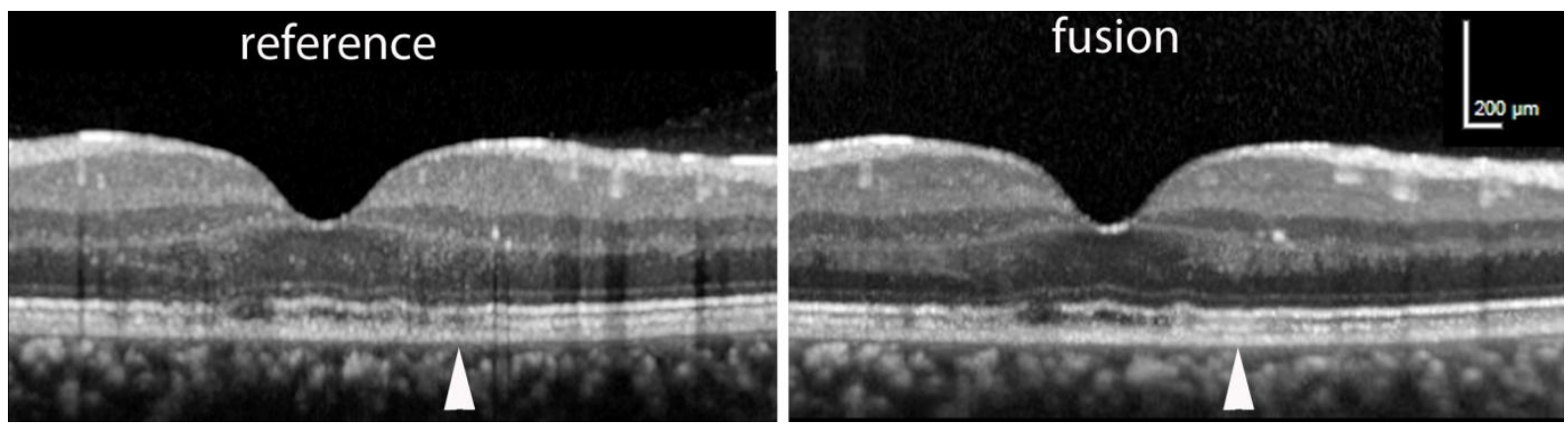

Figure 5. Case 5. Illustration of the partial restoration of the continuity of the IS/OS and COST lines (arrowheads) by combining reference and off-axis scans in a fusion image. 

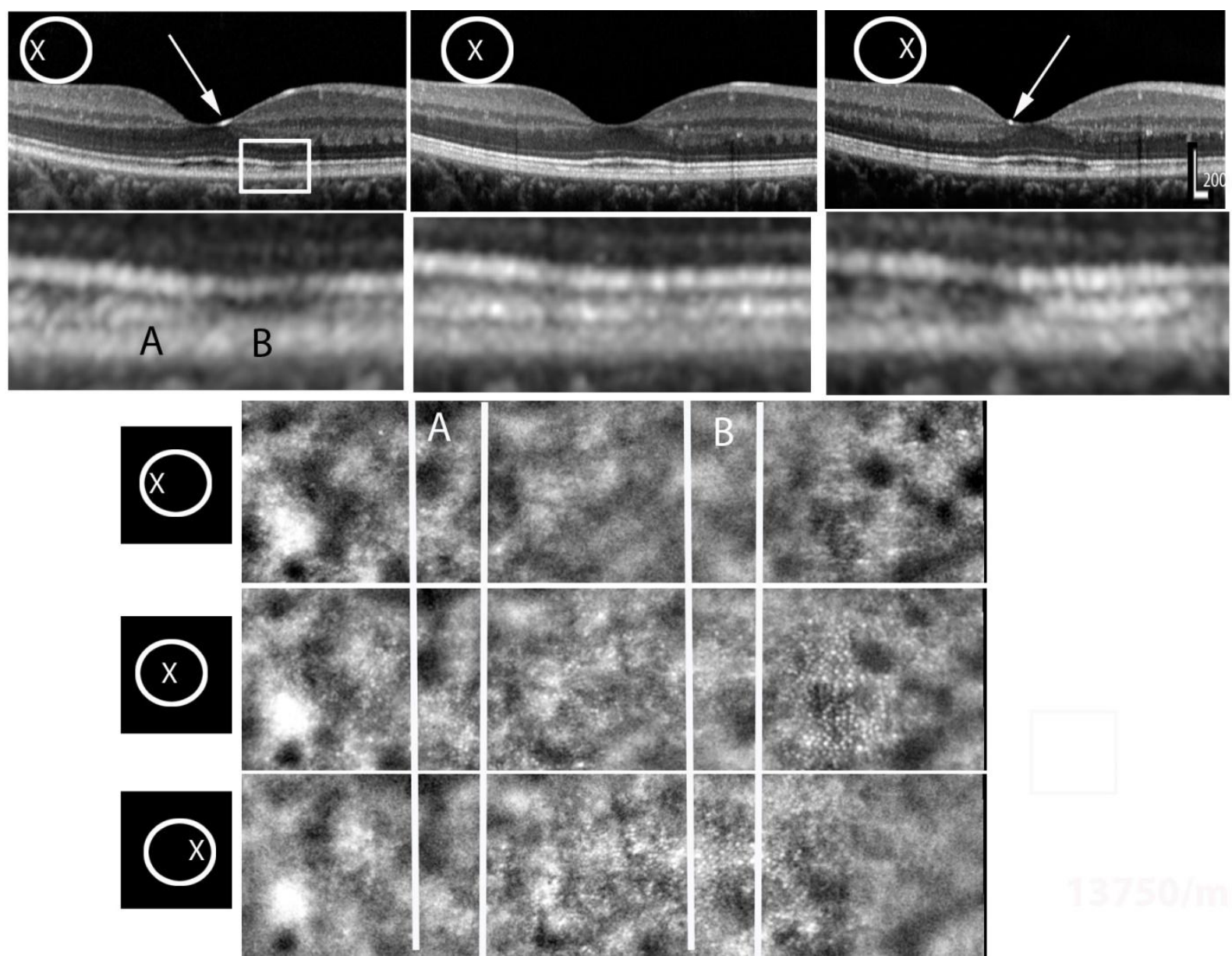

Figure 6. Case 6. Top, DOCT scans with magnification of an ROI showing directional

variability of outer bands. The entry pupil is figured by the $\mathrm{X}$ in the top row. Bottom, magnification of $A O O$ in the same area shown on OCT magnifications. $A$ and $B$ are two zones of the outer retina showing divergent pointing. The variations of the COST intensity matches those of the cone mosaic both in A and B. Note that on the reference (on-axis) scan, outer bands appears continuous; focal attenuation is only detected on off-axis scans. 

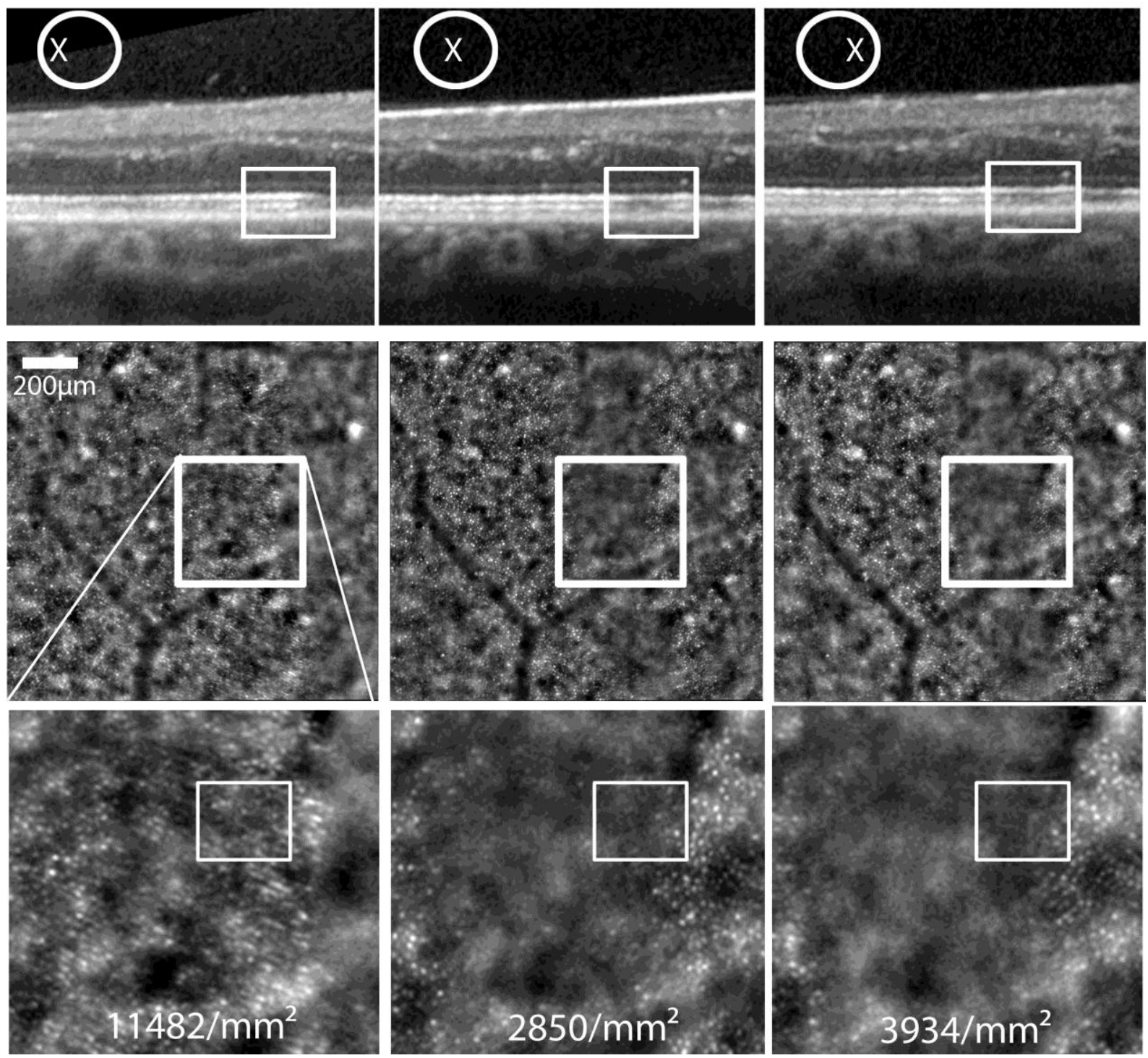

Figure 7. Case 7. Directional variability of cones imaging by OCT (top) and AOO in an area $6^{\circ}$ temporal from the fovea. Each column corresponds to the same entry pupil (figured by the $\mathrm{X}$ in the top row). Note that cones in the center of the image are not visible by $A O O$ on the reference image, while they are visible on the image taken through the left side of the pupil. The cone densities are indicated in the magnifications of $\mathrm{AOO}$ images (bottom row). 


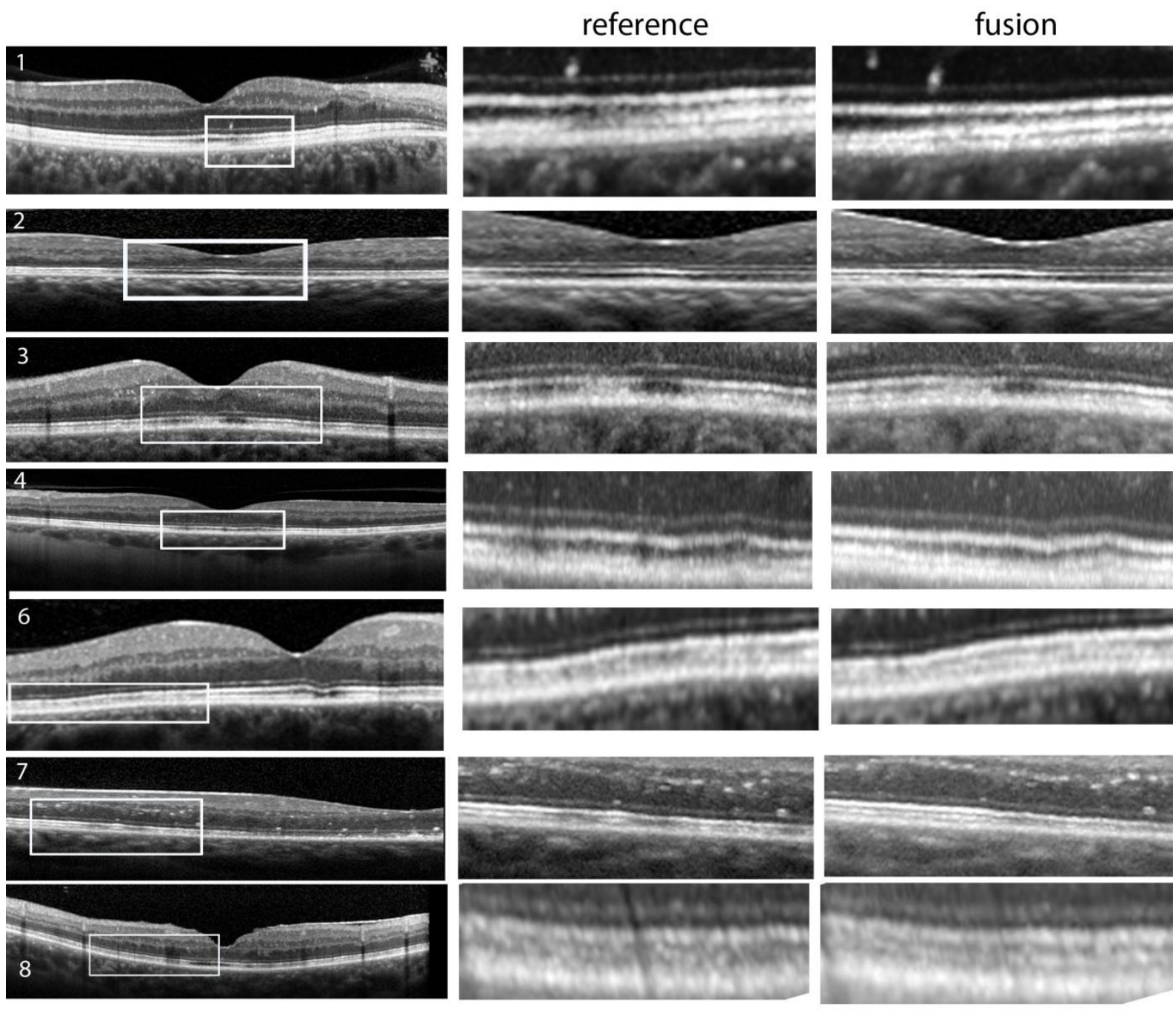

Supplementary figure 1. pdf

Reference and fusion images of 7 cases (right column: magnification of the area in the square). 